\title{
Visual Exploration of HARDI Fibers with Probabilistic Tracking
}

\author{
Ronghua Liang+*, Zhengzhou Wang+, Song Zhang-, Yuanjing Feng+, Li Jiang+, \\ Xiangyin Ma+, Wei Chen\#,David F. Tate\$
}

+College of Information Engineering, Zhejiang University of Technology, Hangzhou, R.R. China, 310023

-Computer Science and Engineering, Mississippi State University, MS, USA,39762

\#State Key Lab of CAD\&CG, Zhejiang University, Hangzhou, P.R.China, 310027

\$University of Missouri, St. Louis, Missouri Institute of Mental Health, Berkeley, MO, USA

*Corresponding Author, Ronghua Liang, Tel. +86-571-85290565, Fax. +86-571-85290565, Email: rhliang@zjut.edu.cn

\section{Abstract}

High angular resolution diffusion imaging (HARDI) is an effective method for characterizing complex neural fiber paths in the human brain. However, visualizing and analyzing the fibers is often challenging because of the complexity of the fiber orientation distribution function used to describe the crossing, kissing, and fanning fibers. In this paper, we propose a novel visual analytics approach to study brain fiber paths that allows users to explore fiber bundles to reveal the probability of fiber paths using a new visual classification method. First, we use a spherical deconvolution model for diffusion estimation and a Bayesian theorem for fiber tractography. Second, each fiber is subjected to a cluster analysis using pixel-based visual encoding. This result is shown in a pixel-based visual representation where each pixel bar maps opacity, color, and length to the probability, direction, and length of a fiber. Fiber bundles can then be acquired via a two-step classification routine that uses DBSCAN to group fibers based on similarities. Then the user can further refine fiber bundle selection using probabilistic information from the pixel bars. Therefore, the proposed approach shows not only the shape but also the confidence of the fiber paths. We demonstrate the resulting HARDI fiber bundles and compare a brain with tumor and normal brain using our system. Experiments and an empirical user study verify the effectiveness of our approach.

Key words: Probabilistic tractography, 3D ellipsoidal glyphs, pixel bar, fiber bundle classification

\section{Introduction}

Diffusion weighted magnetic resonance imaging (DW-MRI) is a noninvasive imaging technique that can reveal the microstructural information of the human brain by measuring the diffusion of water molecules inside biological tissues. To visualize and measure fiber orientation, diffusion tensor imaging (DTI) [3] was proposed for estimating the probability distribution of water 
molecules through the use of 3D Gaussian approximation. DTI can non-invasively map the 3D structure of neural fibers in the brain because the principal eigenvector of the tensor is consistent with the gross fiber orientation in coherent regions [10]. However, more than one fiber orientation (i.e., in crossing or kissing fibers) may exist within a single imaging voxel and simple DTI methods are limited in its ability to recover structures in areas with complex intra-voxel heterogeneity. To address this problem, high angular resolution diffusion imaging (HARDI) techniques were developed to resolve local crossing fibers within a voxel. In HARDI, the orientation distribution functions (ODF) for describing the diffusion profile can have multiple maxima and thus capture complex fiber structures such as crossing, kissing, merging, curving, and fanning fibers.

HARDI diffusion estimation can be grouped into two categories: model-free and model-based approaches. Model-free approaches, also called Q-space techniques, identify multiple fiber components by calculating the probability distribution functions (PDF) of the diffusion process on the basis of the Fourier transform relationship between the PDF of diffusion displacement and the diffusion-weighted signal attenuation in Q-space. Examples of model-free approaches include Q-ball imaging (QBI) [12], diffusion spectrum imaging (DSI) [43], and diffusion orientation transform (DOT) [34]. Model-based approaches apply a model to the data and extract a fiber orientation distribution (FOD). Examples of model-based approaches include multi-tensor model [30], directional functions [37], spherical harmonics deconvolution (SHD) [42][13], and higher order tensor (HOT) [21][48][17].

Tractography is an extension of tensor estimation and provides a method of tracing 3D neural fibers using local fiber orientations [4]. HARDI-based tractography algorithms can be classified as deterministic or probabilistic. Deterministic tracking methods such as streamlines [4] or variations of streamlines [10] in 3D are often used because of their efficient computation. Deterministic tracking methods are limited by their sensitivity to the estimated principal direction and their dependency on the choice of initialization [13]. Therefore, HARDI-based probabilistic algorithms were developed to overcome these drawbacks [16]. However, since errors can accumulate as a fiber is tracked over a long distance, the probabilistic tractography might produce spurious connections though the evaluation and visualization of these potential fiber errors remain largely unexplored [51].

In this paper, we propose a novel visual analysis approach for studying the paths of brain fibers using probabilistic tractography of HARDI data. We adopt a spherical deconvolution (SD) model proposed by Tournier [42] to encode the orientation distribution of fibers at each voxel. Three-dimensional ellipsoidal HARDI glyphs are used to show multiple orientations in voxels, and probabilistic fiber tractography based on Bayesian theorem is then used to track brain fibers. A pixel-based visual encoding that depicts the probability, direction, and length of each fiber is employed for clustering analysis and confidence-based fiber selection. The results of the methods are provided for both artificial and real data and the effectiveness of the results is evaluated by a domain expert. A comparison between a normal brain and a brain with tumor clearly shows the injury to neural fibers around the tumor.

The remainder of the paper is organized as follows. A review of related work is presented in Section 2. The proposed method is discussed in detail in Section 3. The results are depicted in Section 4, and qualitatively evaluated by a neuropsychologist. We summarize the conclusion in Section 5 . 


\section{Related Work}

Tensor glyphs represent the local diffusion information by the shape and orientation of their geometry [35]. For example, cuboids and ellipsoids have been used to show orientations in DTI. Kindlmann et al. [25] adopted super-quadrics to describe Gaussian distribution of fiber in tissues and visualize diffusion tensors with the use of glyph packing techniques. However, three-dimensional glyphs for DTI are typically insufficient for HARDI because of the additional complexity ODF because it is often presented as a symmetric spherical glyph that shows the dominant diffusion directions on the 3D diffusion profile within a voxel. More advanced techniques like Hlawitschka and Scheuermann [31] used GPU ray-casting technique to render superquadric tensor glyphs. Van Almsick [45] defined a new 3D glyph to depict the ODF and implemented it in GPU. Jiao [22] presented a method for constructing a shape inclusion probability function from a series of estimated diffusion shapes for uncertainty analysis. Shattuck et al. [40] developed a mesh polygon on a sphere to obtain the ODF though this method requires complex computation and is time-consuming. In this paper, we introduce 3D ellipsoidal glyphs derived from a spherical deconvolution model to encode the orientation distribution of fibers in a voxel.

Tractography can be used to explore the global connectivity. A number of deterministic methods have been proposed. For example, Van Wedeen et al. [47] proposed a generalized streamline tracking algorithm based on the directions of maximum diffusion that represent local orientations computed from DSI [43]. Chamberland et al.[7] proposed a real-time fiber tracking tool to isolate the parameters which are sensitive to tractography . Ramon Aranda et al. [1] proposed a method that uses the multiple local orientation information to estimate axonal fiber pathways and obtain smooth, filtered and compact trajectory bundles. Assemlal et al. [2] proposed an extension of the streamline to deal with fiber crossings based on the conventional diffusion ODF reconstructed from QBI [12]. However, these methods use the principal direction of the diffusion to track fibers and are thus sensitive to the estimated principal direction.

In contrast, HARDI-based probabilistic tractography techniques [18] incorporate the uncertainty of the principal diffusion direction in the tracking process, thus overcoming many of the limitations of deterministic tracking. Jbabdi et al. [18] used a Bayesian framework to perform global tractography instead of tracking through local orientations. The output of probabilistic fiber tracking can produce a connectivity index that measures the probability that two voxels are connected to each other. These methods usually require a large number of samples. Friman et al. [16] used 10,000 seeding points per voxel, but it was found that most did not reach the desired region. Zhang et al. [19] used 1000-5000 particles with improved results. Other robust Bayesian modeling techniques that combine mixture models [5] and parametric spherical deconvolution [23] have been proposed. Manginet al.[29] described an overview of state-of-arts achievements of global tractography and proposed guidelines for a future research of probabilistic tractography methods.

Many approaches have been proposed to address uncertainty visualization [36][27] Error!

Reference source not found.[9]. However, they seldom consider the uncertainty in probabilistic tractography. V. Wiens et al.[49] used superquadric streamtubes to visualize uncertainty in the tracking direction of HARDI tractography. However, this visualization relies on fine shape details on the streamtubes to encode uncertainty making it prone to visual clutters with dense sampling. 
Thus, fiber paths are still difficult to track and analyze in the context of probabilistic tractography.

The main objective of fiber clustering is to group similar fibers into bundles generally on the basis of their similarities in position and shape [50], e.g., the Euclidean distance or Hausdorff distance between fibers. Clustering is an important tool for creating abstractions and filtrations in general and is central to our representation as successful tract clustering can capture anatomical features valuable to the users. Several approaches, such as Open-Box Spectral Clustering [39] or $3 \mathrm{D}$ to $2 \mathrm{D}$ mapping [8][20], use 3D space clustering. Wang et al. [46] proposed a fiber clustering method that relies on the neuroanatomical information of each fiber.

Building on these methods, we present a novel approach for clustering fiber bundles by introducing a pixel bar to represent the fibers. Every pixel in a bar is associated with an equally spaced point in a fiber. Its color is associated with the orientation of the voxel; its opacity represents the overall probability of the fiber; and the length of bar is associated with the length of the fiber. Users can then produce a fiber bundle by choosing the specific opacity value on pixel bars, thus exploring the fibers within the user-defined confidence levels.

\section{Method}

\subsection{Algorithm outline}

Our visualization pipeline is shown in Figure 1. At the first stage, a spherical deconvolution(SD) model for fiber orientation estimation is solved by a linear system and recovered with the spherical Gaussian kernel. We use a 3D ellipsoidal glyph to decode the solution of each voxel in 3D space. At the second stage, Bayesian probabilistic fiber tracking is adopted to obtain the pathway of each fiber. To reveal the uncertainty that exists in probabilistic tracking, we use a pixel bar to encode each fiber with orientation, probability, and length. Relying on the visual information provided in the pixel bar, any unlikely results could be eliminated according to the user's selection.

In order to validate the effectiveness of our approach, we render the fibers of a whole brain with 100 possible directions per voxel. With this method, the whole brain fibers are produced and explored. The visual results for a brain tumor patient are shown to illustrate how easy it is to visualize the change of fibers around a tumor in the brain.

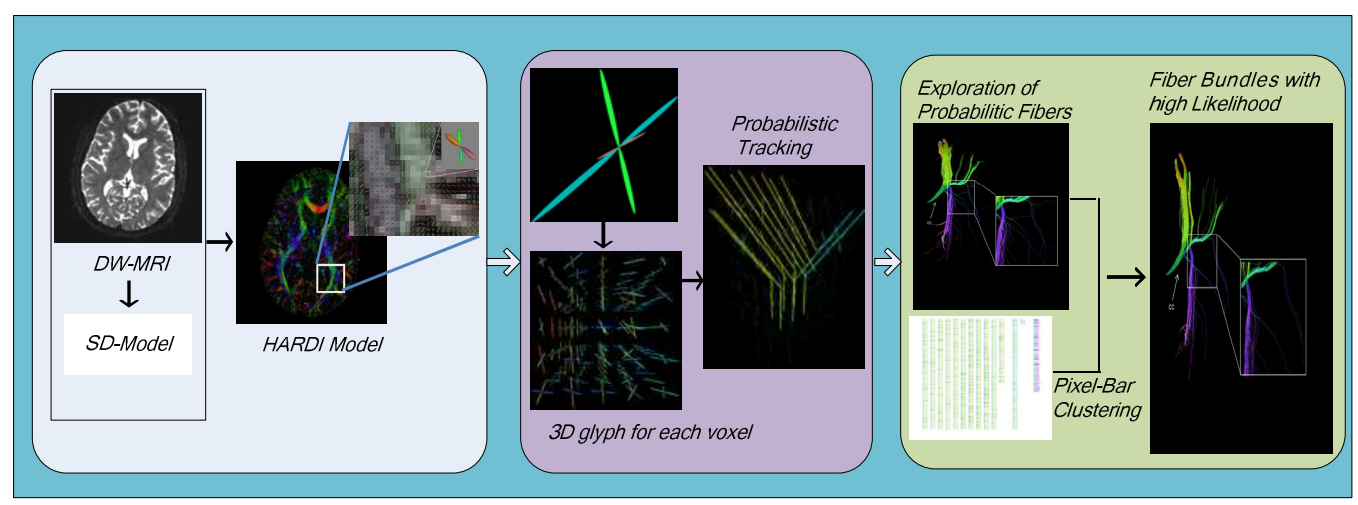

Fig. 1. Our visualization pipeline for fiber probabilistic tracking 


\subsection{Diffusion model estimation}

We estimate the spherical deconvolution model by solving a linear system and recovering with the spherical Gaussian kernel. In the spherical deconvolution model [42], the diffusion signal $\mathrm{S}(\mathrm{g}) / \mathrm{S}_{0}$ with $N$ gradient directions $g$ is generally approximated as the convolution of the response function $\mathrm{R}(\mathrm{g}, \hat{\mathrm{x}})$ with the fiber orientation distribution function (fODF) $F(\hat{\mathrm{x}})$ over the unit sphere, as expressed below:

$$
S(\boldsymbol{g}) / S_{0}=F(\hat{\boldsymbol{x}}) \otimes R(\boldsymbol{g}, \hat{\boldsymbol{x}})
$$

where $\hat{x}$ is the convolution sample directions. After setting the response function as $R(\mathrm{~g}, \hat{\mathrm{x}})=e^{-\delta(\mathrm{g} \cdot \hat{\mathrm{x}})^{2}} \quad(\delta$ is the product of diffusion time and diffusivity), we can obtain the fODF $F(\hat{\mathrm{x}})$ by performing the spherical deconvolution of $R(\mathrm{~g}, \hat{\mathrm{x}})$ from $S(\mathrm{~g}) / \mathrm{S}_{0}$. As a sum of $M$

Dirac delta functions, $F(\hat{\mathrm{x}})$ can be estimated by minimizing the following energy as

$$
E=\sum_{i=1}^{N}\left(S\left(\boldsymbol{g}_{i}\right) / S_{0}-\sum_{j=i}^{M} e^{-\delta\left(\boldsymbol{g}_{i} \cdot \hat{\boldsymbol{x}}_{j}\right)^{2}} F_{j}(\hat{\boldsymbol{x}})\right)^{2}
$$

which is expressed as a linear system and can be solved by the non-negative least squares technique. Finally, to prevent the influence of noise, we adopted the spherical Gaussian kernel instead of the spherical harmonic [42] to recover each $F_{j}(\hat{\mathrm{x}})$ into the recovered fODF $f(\hat{\mathrm{x}})$

$$
f(\hat{\boldsymbol{x}})=F(\hat{\boldsymbol{x}}) \cdot G(\boldsymbol{g}, \hat{\boldsymbol{x}})
$$

where $G(\mathrm{~g}, \hat{\mathrm{x}})=\exp \left(-\cos ^{-1}\left((\mathrm{~g}, \hat{\mathrm{x}})^{2} / \sigma^{2}\right)\right), \sigma$ is the width parameter, which is numerically configured to prevent insufficient merging or decreases in angular resolution. With this ODF model, each voxel can have more than one orientation, and it can be used for distinguishing the crossover nerve fiber tracts.

We use 3D ellipsoidal glyphs to represent the orientation distribution in each voxel. Ellipsoidal glyphs $\mathrm{G}(\mathrm{Q})$ are aligned with the eigenvectors of the tensor at a position $\mathrm{Q}$. There are at most three pairs of ellipsoidal glyphs to represent at most three fiber orientations inside a voxel. Figure 2 shows the ellipsoidal glyphs at multiple level-of-details for an artificial data set described in Section 4.1. 


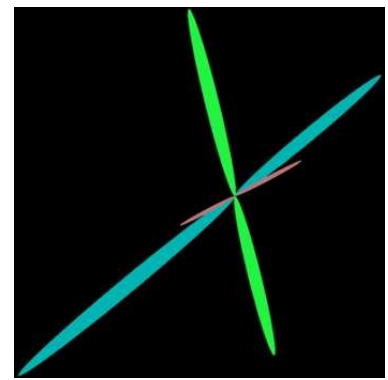

(a)

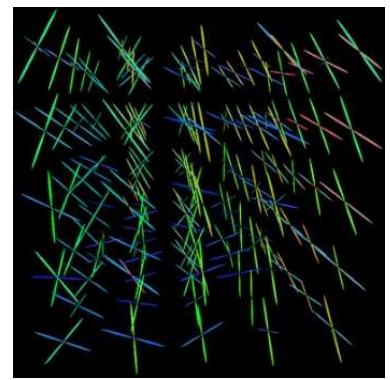

(d)

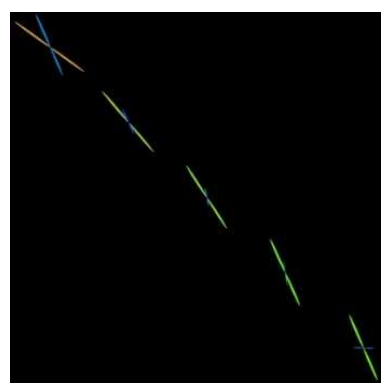

(b)

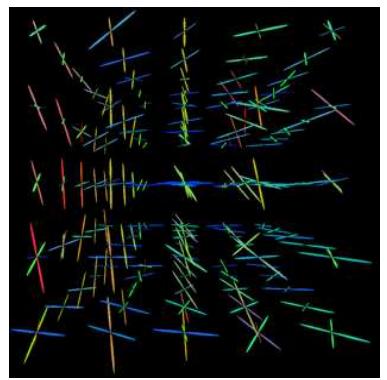

(e)

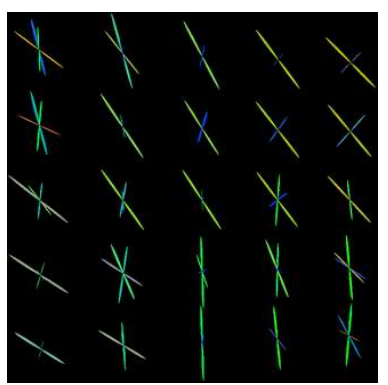

(c)

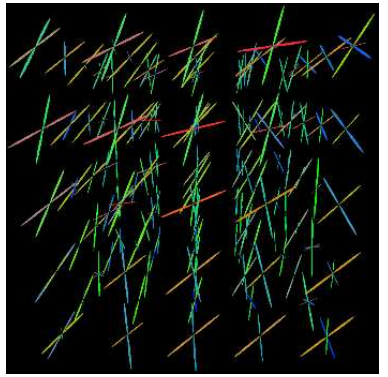

(f)

Fig. 2. 3D ellipsoidal glyphs representation of the diffusion model: (a) glyphs for a single voxel; (b) glyphs for a few voxels along a fiber; (c) glyphs for voxels in a $5 \times 5 \times 1$ grid; (d), (e), and (f) three different views of glyphs in a $5 \times 5 \times 5$ grid;

\subsection{Probabilistic fiber tracking}

We pose the fiber tracking as a Bayesian decision problem as shown in Error! Reference source not found.. For each step, the direction $v_{i}$ is sampled from a probabilistic distribution function

(PDF) $p\left(\mathrm{v}_{i} \mid \mathrm{v}_{i-1}, \Phi\right)$, which is expressed by a Bayesian framework [15],

$$
p\left(\boldsymbol{v}_{i} \mid \boldsymbol{v}_{i-1}, \Phi\right)=p\left(\Phi \mid \boldsymbol{v}_{i}\right) p\left(\boldsymbol{v}_{i} \mid \boldsymbol{v}_{i-1}\right) / p(\Phi)
$$

where $\Phi$ is the set of observations of a 3D diffusion weighted imaging volume. $p(\Phi)$ is a fixed normalization factor of the system as in [6]. The priori distribution $p\left(\mathrm{v}_{i} \mid \mathrm{v}_{i-1}\right)$ that defines priori knowledge is given by a simple distribution family [10]. The observation model $p\left(\Phi \mid \mathrm{v}_{i}\right)$ is substituted as the fODF in (3)

$$
p\left(\Phi \mid v_{i}\right)=f\left(v_{i}\right)
$$

Thus, the PDF $p\left(\mathrm{v}_{i} \mid \mathrm{v}_{i-1}, \Phi\right)$, or the posterior distribution, is computed from Eq. (4) when the priori and observation densities are both calculated. Finally, we utilize MCMC technique [26] to draw a random sample of the fiber direction.

In a whole brain, neural fibers are very complex because crossing, merging, and splitting 
fiber tracts are all present. In HARDI data, the ODF per voxel has different directions and thus produces different pathways. Figure 3 shows the local structure with ellipsoidal glyphs and the reconstructed pathways with lines. Figure 3 (a) shows a $5 \times 5 \times 5$ grid data in a real brain, and Figure 3 (b) shows pathways in a $3 \times 4 \times 5$ grid artificial data described in Section 4.1.

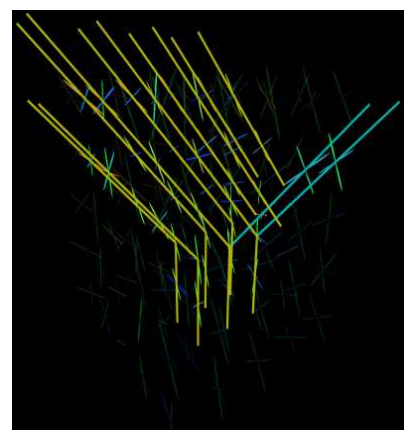

(a)

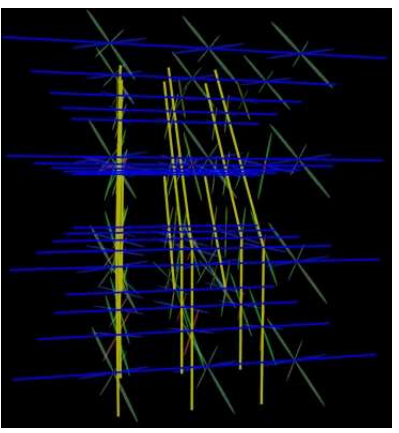

(b)

Fig. 3. Fiber tracking with our method in two datasets: (a) a $5 \times 5 \times 5$ grid real brain data and (b) a $3 \times 4 \times 5$ grid artificial data.

\subsection{Fiber generation with high likelihood}

Fiber pathways can be acquired with the aforementioned probabilistic tracking. However, to show the inherent uncertainty in probabilistic tracking, these fibers must be presented in the context of their uncertainty. In this section, we present a novel visual analysis method for fiber path classification, and our approach adopts pixel-based visual encoding for the direction, probability, and length of each fiber. We also allow users to interact with the 2D pixel-based visual representation to cluster the fiber bundles based on their uncertainty.

Pixel bar mapping. A 2D pixel bar [24] is used to map a 3D fiber. In the pixel bar, the color encodes the orientation, the length encodes the actual length of the fiber, and the transparency represents the overall probability of the fiber. An orientation is represented by a vector $\vec{n}(x, y, z)$. To ensure the correct range in the RGB color space, we normalize the vector and map it to RBG values as follows:

$$
\left\{\begin{array}{l}
\mathrm{R}=1 / 2 *\left(1+\mathrm{x} / \sqrt{\mathrm{x}^{2}+\mathrm{y}^{2}+\mathrm{z}^{2}}\right) * \mathrm{R}_{\max } \\
\mathrm{G}=1 / 2 *\left(1+\mathrm{y} / \sqrt{\mathrm{x}^{2}+\mathrm{y}^{2}+\mathrm{z}^{2}}\right) * \mathrm{G}_{\text {max }} \\
\mathrm{B}=1 / 2 *\left(1+\mathrm{z} / \sqrt{\mathrm{x}^{2}+\mathrm{y}^{2}+\mathrm{z}^{2}}\right) * \mathrm{~B}_{\max }
\end{array}\right.
$$

where $R_{\max }=G_{\max }=B_{\max }=255$. A hue ball shown in Figure 4(a) demonstrates how the color encodes the orientation.

The orientation probability for each voxel $\mathrm{P}_{\mathrm{i}}$ in Bayesian tracking is calculated by a fixed normalization factor and prior knowledge described in Section 3.3. The $\mathrm{P}_{\mathrm{MSE}}$ of each fiber is expressed by MSE, i.e., $\mathrm{P}_{\mathrm{MSE}}=\sqrt{\sum_{\mathrm{k}=1}^{\mathrm{n}}\left(\mathrm{p}_{\mathrm{i}}-\overline{\mathrm{p}}\right)^{2} / n}$, where $\overline{\mathrm{p}}$ is the average of the orientation probability of all voxels within a fiber and $n$ is the number of voxels. $P_{\mathrm{MSE}}$ denotes the fluctuation of the probability within a fiber, and lower $\mathrm{P}_{\mathrm{MSE}}$ corresponds to fibers that have a consistent probability along its arc length. The value $\mathrm{P}_{\mathrm{MSE}}$ can be directly mapped into the transparency $\alpha$ of a pixel bar. Figure 4(b) shows an example of a pixel bar for a fiber. 


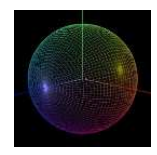

(a) hue ball

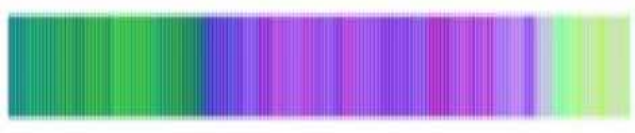

(b) a pixel bar

Fig. 4. Pixel bar representation of a fiber in 3D space: (a) a hue ball encodes orientation with color; (b) in a pixel bar, color encodes the orientation of each voxel in a fiber; pixel-bar length is mapped to the length of the fiber, and the transparency encodes the uncertainty of a fiber.

Fiber bundles classification. Using Bayesian tracking, we can obtain numerous fibers from a seeding point (see Figure 5 (a)). However, empirical studies indicate that most fibers have low probability and are therefore not desirable. The fibers can be encoded using the pixel bar information, as shown in Figure 6 (a). In general, 2D rather than 3D interactive operations are easier for the users. Therefore we adopt a two-step approach to analyze the probabilistic fibers. The first step is the automatic classification of the pixel bars into bundles. The second step is for the user to choose a threshold on the fiber bundle and eliminate fibers whose $\mathrm{P}_{\mathrm{MSE}}$ values exceed this threshold.

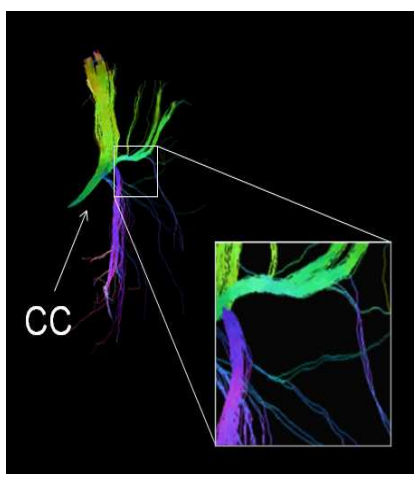

(a)

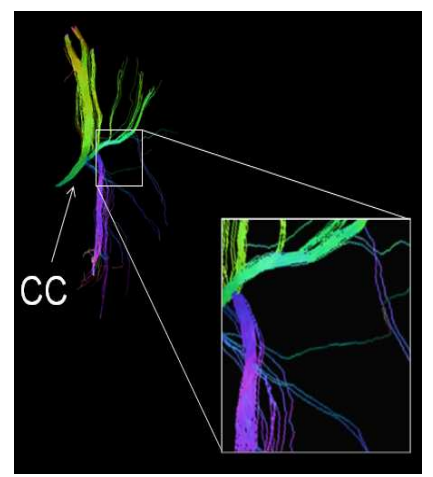

(b)

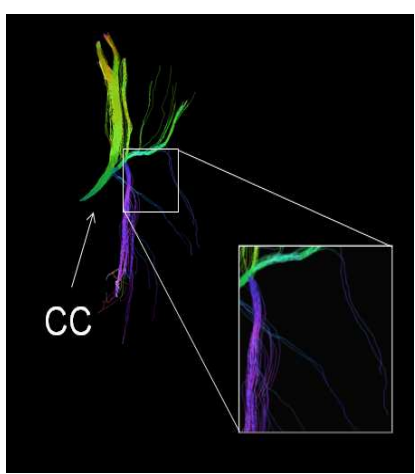

(c)

Fig. 5 Fiber paths in Corpus Callosum (CC) with a different $\mathrm{P}_{\mathrm{MSE}}$ value. A lower $\mathrm{P}_{\mathrm{MSE}}$ value produces a higher likelihood of fiber bundle. (a) Fibers are produced by probabilistic tractography without user-selection. (b) Fibers are selected with $\mathrm{P}_{\mathrm{MSE}}<0.4$. The bright green fiber bundles at top left corner of the close-up view in (b) are sparser compared with the same region in (a). (c) Fibers are selected with $\mathrm{P}_{\mathrm{MSE}}<0.35$.

For automatic cluster classification, we employ a DBSCAN [14] seeding points method on pixel bars to cluster these fibers. This may be desirable because DBSCAN is efficient for clustering high-density data like the fibers from probabilistic tractography. DBSCAN have two user-defined parameters, namely, scan radius and density threshold, which can be adapted to pixel bar classification. In our implementation, scan radius is empirically set to 2.5 and density threshold is set to 20 , meaning that the pixel bars within 2.5 will be classified into one group, and fibers with less than 20 fibers in the radius of 2.5 are deemed to be not part of any group. We define the distance between two pixel bars with $\mathrm{N}$ and $\mathrm{M}$ points:

$$
\mathrm{D}=\frac{\sum_{\mathrm{i}=1}^{\mathrm{n}} \mathrm{d}_{\mathrm{i}}}{1 / 2(\mathrm{~N}+\mathrm{M})}
$$

where $\mathrm{n}=\min (\mathrm{N}, \mathrm{M}), \mathrm{d}_{\mathrm{i}}$ is the Euclidean distance between the $i$ th points along two fibers. After DBSCAN clustering, every fiber within one cluster meets the requirement that the distance 
between it and any other fiber in the cluster is less than the scan radius. Those fibers with density less than the threshold are considered unlikely. An example of DBSCAN clusters are shown in Figure 6.

Utilizing this visual information, the user can interact with the pixel bars (see Figure 6) and choose the fiber bundles with high likelihood (shown in Figure 5(b)). Figure 5 illustrates the potential benefits of choosing fibers as fibers with high $\mathrm{P}_{\mathrm{MSE}}$ have abnormal directions, whereas fibers with low $\mathrm{P}_{\mathrm{MSE}}$ have coherent directions.

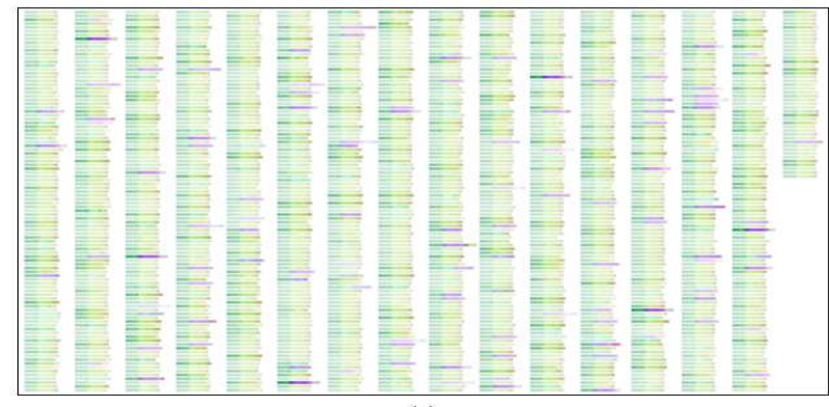

(a)

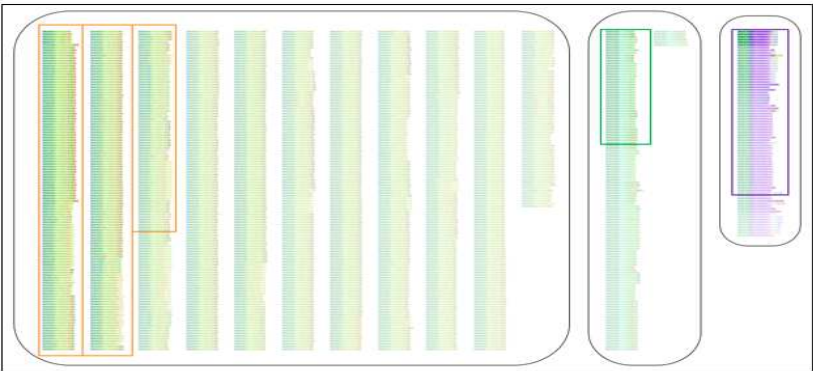

(b)

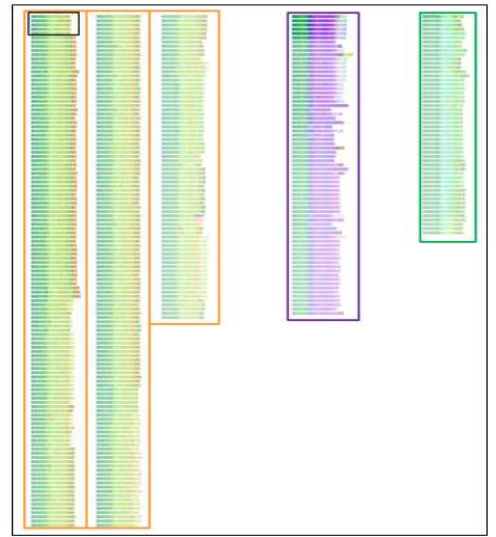

(c)

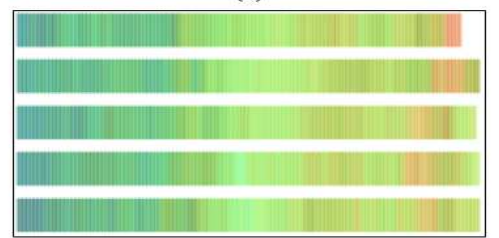

(d)

Fig. 6 Pixel bar with representation of fibers in Fig. 5. (a): pixel bars for fibers in Fig 5 (a) before clustering. (b): pixel bars associated with the three clusters in Fig. 5(a) after DBSCAN clustering. In each cluster, pixel bars are sorted by transparency. Users can make confidence-based selection of the fibers in a bundle (orange, green, and purple rectangle). (c): A close-up view of the user-selected fibers in (b). (d): A close-up view of the black rectangle box in (c).

\section{Experiments}

\subsection{DW-MRI data}

The DWI dataset of a normal human brain was acquired with a 3T GE system and a double echo planar imaging (EPI) sequence, with TR $=17000 \mathrm{~ms}$ and $\mathrm{TE}=78 \mathrm{~ms}$. The images cover a field of view of $24 \mathrm{~cm}$ with a $144 \times 144$ grid. A total of 85 axial slices parallel to the AC-PC line were acquired, with a slice thickness of $1.7 \mathrm{~mm}$. The acquisitions had 51 gradient directions with $\mathrm{b}=$ $900 \mathrm{~s} / \mathrm{mm}^{2}$ and 8 baseline scans with $\mathrm{b}=0$.

The DWI dataset of a human brain with tumor was acquired from a male adult on a 3T MRI system (TIM Trio, Siemens, Erlangen, Germany). The spatial parameters were fixed as follows: matrix size $=256 \times 256 \times 50$. The acquisitions had 51 diffusion gradient directions with $b=2000$ $\mathrm{s} / \mathrm{mm}^{2}$.

The artificial data were generated by simulating the Magnetic Resonance (MR) signals using 
the diffusion MR simulation model:

$$
\mathrm{S}(\mathrm{g})=\sum_{\mathrm{i}=1}^{\mathrm{n}} \mathrm{f}_{\mathrm{i}} \mathrm{e}^{-\mathrm{bg}^{\mathrm{T}} \mathrm{Dg}}
$$

where $f_{i}$ represents the fraction contribution of the $i$ fiber. The diffusion tensor $D$ for each fiber was calculated with eigenvectors oriented along the desired directions and eigenvalues are as

follows: $\lambda_{1}=1.7 \times 10^{-3} \mathrm{~mm}^{2} / \mathrm{s}, \quad \lambda_{2}=\lambda_{3}=0.2 \times 10^{-3} \mathrm{~mm}^{2} / \mathrm{s}, \mathrm{b}=2000 \mathrm{~s} / \mathrm{mm}^{2}$. A total of 81 uniformly distributed gradient directions were obtained from the twofold icosahedron tessellation. The selected number for convolution sample directions is 321, which was derived from the threefold icosahedron tessellation. Rician noise was added to the simulated signal datasets.

\subsection{Results}

We have implemented a prototype of our method in C++ using OpenGL and QT libraries on the computer with Inter Xeon CPU @2.27GHz, NVIDIA Quadro FX 5600.

Figure 5 shows the results of probabilistic fiber tracking from one seeding point. Fibers are shown in Figure 5, whereas the pixel bars in Figure 6 show the distribution of key properties of these fibers for user analysis, grouping, and selection.

Figure 7 shows two bundles (Ventral Transverse Pontine and Corticospinal Tracts) of fibers produced by a seeding point. Fibers are selected using different $\mathrm{P}_{\mathrm{MSE}}$ values depicted in Figures 7(b)(c) and Figures 7 (e)(f).

Figure 8 shows the comparison between a previous fiber clustering algorithm [8] and our proposed method. The clustering algorithm in Figures 8(a) is based on two-dimensional MDS with k-means clustering. Partition-based k-means method is performed with the two-dimensional MDS (the scatter diagram at the bottom of (a)). Hence, corresponding fibers in three dimensions seem to be completely mixed together. However, our seeding points related classification method is based on spatial distance, and the clusters are clearly distinguished in Figure 8(b). In addition the white fibers in (b) are eliminated as data noise, and they do not belong to any clusters, whereas the algorithm in [6] does not remove the data noise.

Figure 9 shows four views of the whole brain fibers using illumination and anti-aliasing methods. The seeding points are sampled on a $256 \times 256 \times 256$ grid. The orientations of the fibers are encoded with color as presented in Figure 4(a). Major fiber bundles such as the corpus collosum, cingulum bundles, and corona radiata can be clearly identified. In these figures, fine features such as diverging and crossing fibers can be identified. In our data with 51 gradient directions, the visualization can show crossing fibers of small angles up to 35 degree. These features cannot be correctly identified in a DTI dataset.

To validate the effectiveness of our approach, we render the fibers of a whole brain. We obtain the probabilistic fibers with the tractography method described in Section 3.2. 1000 streamline fibers were generated per voxel. For each fiber, a direction was first drawn from the local direction distribution at the seed point. We then moved $0.5 \mathrm{~mm}$ along the sampled direction to a new position. This process is repeated until the fiber goes out of bound or other stop criteria are met. For a region of $\mathrm{n}$ voxels, $1000 \times \mathrm{n}$ fibers were sampled. We use the FreeSurfer tool (https://surfer.nmr.mgh.harvard.edu/) to draw a region of interest [38].

Figure 10 shows the brain with a tumor. Our proposed method shows the breaking points and the deformation of the fibers around the tumor, which can help locating the tumor in the brain. 


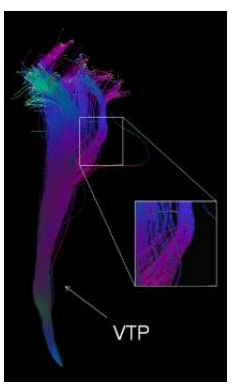

(a)

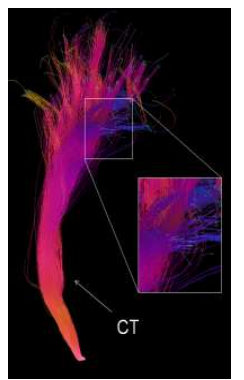

(d)

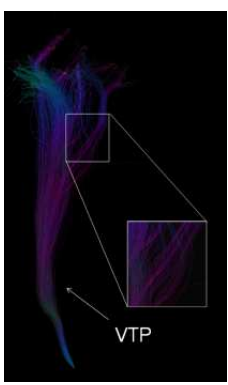

(b)

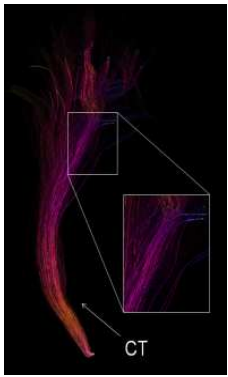

(e)

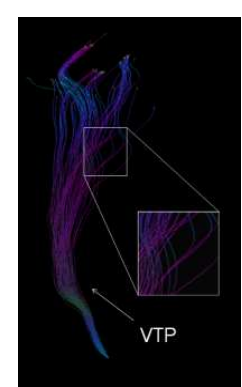

(c)

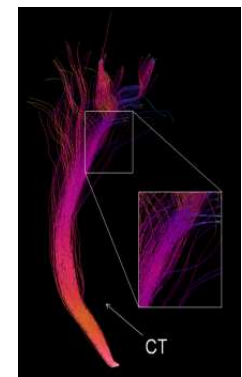

(f)

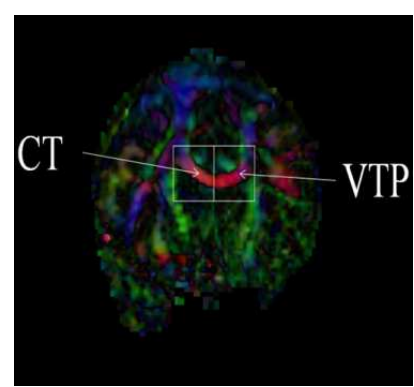

(g)

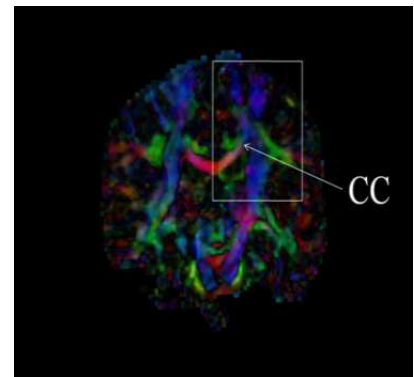

(h)

Fig. 7. Two fiber paths produced by a seeding point. (a) Fibers tracking from a seeding point for Ventral Transverse Pontine (VTP). (b)(e) Fibers selected by $\mathrm{P}_{\mathrm{MSE}}<0.5$. (c)(f) Fibers selected by $\mathrm{P}_{\mathrm{MSE}}<0.35$. (d) Fibers tracking from a seeding point for Corticospinal Tracts (CT) $(\mathrm{h})(\mathrm{g})$ Location of associated regions produced by 3D slicer with the DW-MRI data.

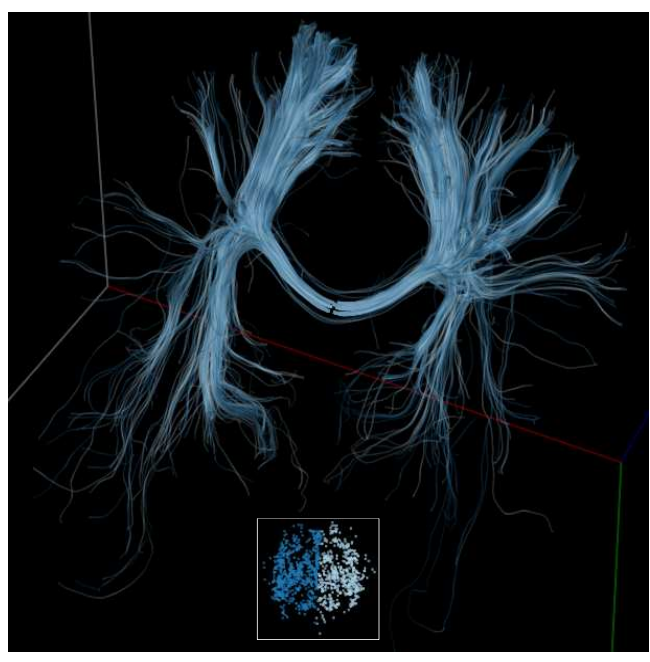

(a)

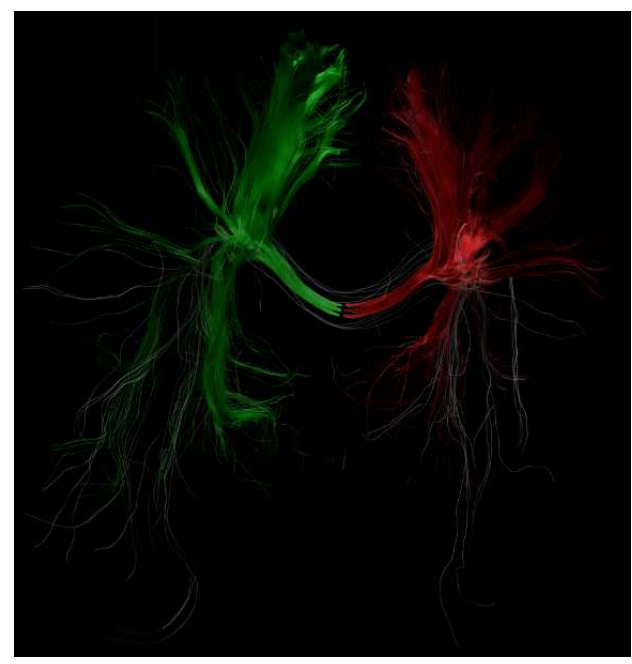

(b)

Fig. 8. A comparison between our approach and method in [8].(a) Fibers are classified into two clusters in blue and white color.(b) Our spatial clustering method classified fibers into two clusters in red and green color. The white fibers in (b) are considered as data noise, they do not belong to any cluster. 


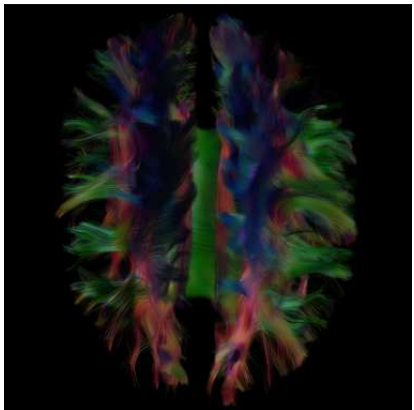

(a)

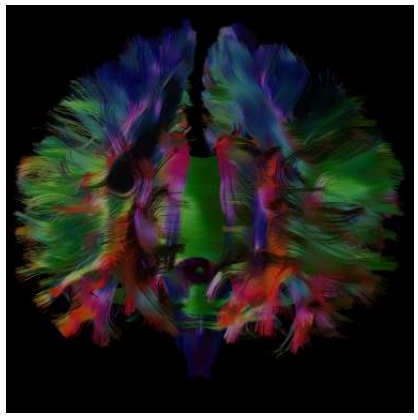

(c)

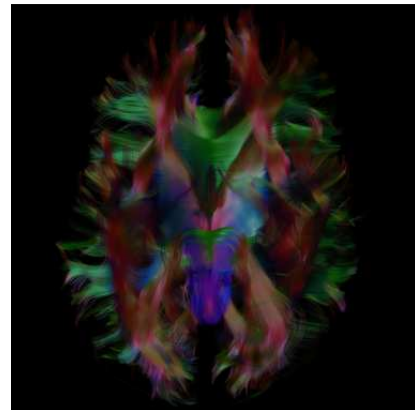

(b)

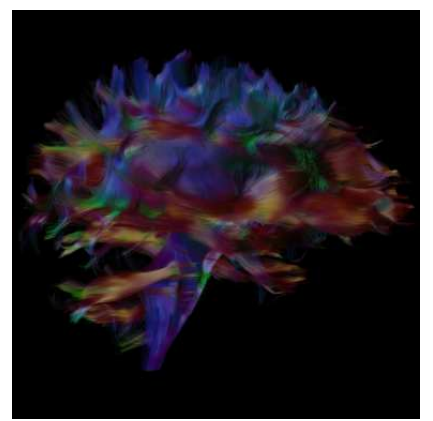

(d)

Fig. 9. Four views of the brain fibers: (a) top view; (b) bottom view; (c) front view; and (d) side view

Probabilistic tracking produces numerous fibers. To show the highly confident fibers, users select on-demand MSE in the pixel bar, and the system can obtain the classified fiber bundles. Table 1 shows the number in the same clustered fiber bundles with different $\mathrm{P}_{\mathrm{MSE}}$. As shown in the table, a low $\mathrm{P}_{\mathrm{MSE}}$ value produces fiber bundles with higher likelihood. However, the validation depends on the user's a priori knowledge.

Table. 1. Number of three clustered fiber bundles with different $\mathrm{P}_{\mathrm{MSE}}$

\begin{tabular}{cccccc}
\hline Data & $0<P_{M S E}<1$ & $P_{M S E}<0.25$ & $P_{M S E}<0.2$ & $P_{M S E}<0.15$ & $P_{M S E}<0.1$ \\
\hline CC & 1544 & 1119 & 786 & 449 & 370 \\
\hline CT & 1083 & 800 & 590 & 352 & 231 \\
\hline VTP & 960 & 531 & 256 & 101 & 67 \\
\hline
\end{tabular}

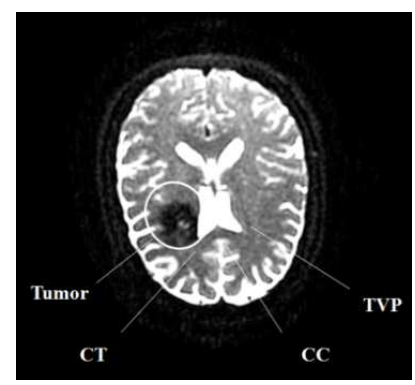

(a)

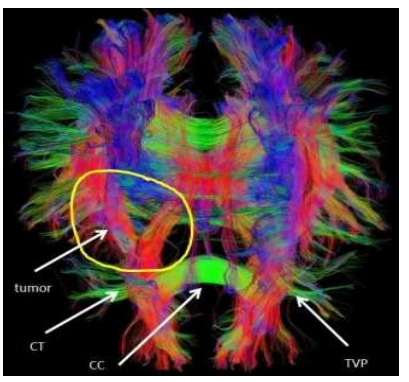

(b) 


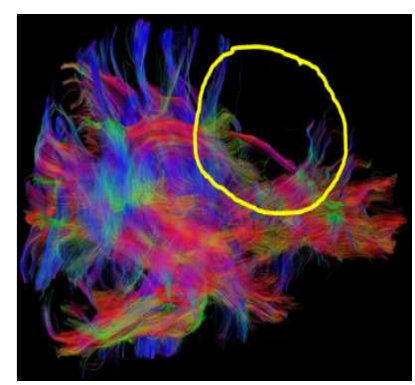

(c)

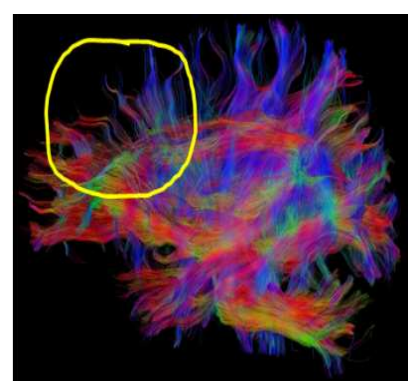

(d)

Fig. 10. Comparisons of fiber connections with a tumor in the same brain: (a) T2-weighted structural image showing tumor; (b) top view of fibers with a tumor; (c) right half of the brain with a tumor; and (d) left half of the brain without tumor

\subsection{Empirical user study}

To evaluate the strength and weakness of our system and gather feedback for method improvement, a neuropsychologist and a diffusion imaging researcher used our visual exploration system and provided the following qualitative observations. A quantitative user study is planned for future work.

(1) Probabilistic fibers capture the main structure of the brain. In Figure 8, the main anatomical structures in the brain, such as the corpus callosum, cingulum bundles, corticospinal tracts, forceps major, forceps minor, and so on, are clearly visible. However, a number of the fibers in the whole brain take unlikely paths compared to similar visualization with diffusion tensor imaging.

(2) With 51 gradient directions in the data, the visualization can show crossing fibers of small angles up to 35 degree. With more gradient directions, smaller angle crossing fibers can be identified. Figure 5 shows all probabilistic fibers from a seed point in corpus callosum. The branching and crossing fibers are clearly shown in the fiber bundle. A close up view of the branching fibers can be observed in Figure 3(a). In comparison, diffusion tensor imaging shows only one orientation at any location. Further study is needed to verify whether these crossing fiber structures indicate realistic anatomical structures.

(3) The visualization system allows the user to apply a priori knowledge of human anatomy and connections in selecting the most likely fibers. This approach is particularly important as it allows users to explore the complex structure of HARDI fibers and the probability of possible tracts generate. However, a balance must be made between the need for additional details and the need for high likelihood especially in disorder that may have heterogeneous pathological effects of spatially unique regions of the brain (i.e., traumatic brain injury).

(4) The user-selected fibers might differ from the automatically generated high likelihood fibers. This phenomenon happens when a user applies a priori knowledge of anatomy to fiber selection that is not encoded in the automatic algorithms and indicates the value of user interaction in HARDI fiber exploration. However, caution would be required as manual intervention could also lead to elimination of relevant pathological features that might be unique to a particular disease or injury.

(5) The pixel bars are intuitive to use and easy to interact with. The visual mapping of the pixel bar, including the length, color, and transparency, are easy to understand and easy to observe 
in visual analysis. Pixel bar representation improves the visual interactivity of the data.

\section{Conclusion}

In this paper, we propose a novel interactive visual analysis approach for studying the connection of brain fiber bundles in the context of uncertainty tractography. We introduce a new 3D glyph derived from a spherical deconvolution model to encode the orientation distribution of fibers at a voxel. We then use probabilistic fiber tractography based on Bayesian theorem to track brain fibers. A pixel-based visual encoding that depicts the probability, direction, and length of each fiber is employed for clustering analysis. This method offers fiber connection detection with high confidence. Expert evaluation confirmed the following: (1) the HARDI fibers have higher fidelity for small angles up to 35 degree crossing fibers in our data; (2) the pixel bar provides an additional useful means of interacting with probabilistic fiber analysis, and (3) probabilistic fiber visualization can effectively assimilate expert knowledge into fiber configuration.

\section{Acknowledgments}

This work was partly supported by National Science Foundation of China (NSFC) 61379076 and 61202205, Program for New Century Excellent Talents in University of China NCET-12-1087.

\section{References}

[1] R. Aranda, M. Rivera, A. Ramirez-Manzanares, A flocking based method for brain tractography, Medical Image Analysis 18 (2014) 515-530.

[2] H. Assemlal, D. Tschumperlé, L. Brun, Fiber Tracking on HARDI Data Using Robust ODF Fields, In: IEEE International conference on Image Processing, 2007,pp. 344-351.

[3] P.J. Basser, J. Mattiello, D. LeBihan, MR diffusion tensor spectroscopy and imaging. Biophysical journal 66 (1994) 259-267.

[4] P.J. Basser, S. Pajevic, C. Pierpaoli, J. Duda, A. Aldroubi, In vivo fiber tractography using DT-MRI data, Magnetic Resonance in Medicine 44 (2000) 625-632.

[5] T.E. Behrens, H. Johansen-Berg, S. Jbabdi, M.F. Rushworth, M.W. Woolrich, Probabilistic diffusion tractography with multiple fibre orientations. What can we gain?, NeuroImage 34 (2007) 144-155.

[6] T. Behrens, M. Woolrich, M. Jenkinson, H. Johansen-Berg, R. Nunes, S. Clare, P. Matthews, J. Brady, S. Smith, Characterization and propagation of uncertainty in diffusion-weighted MR imaging, Magnetic Resonance in Medicine 50 (2003) 1077-1088.

[7] M. Chamberland, K. Whittingstall, D. Fortin, D. Mathieu, M. Descoteaux, Real-time multi-peak tractography for instantaneous connectivity display, Front Neuroinform (2014) 1-15.

[8] W. Chen, Z. Ding, S. Zhang, A. MacKay-Brandt, S. Correia, H. Qu, J.A. Crow, D.F. 
Tate, Z. Yan, Q. Peng, A novel interface for interactive exploration of DTI fibers, IEEE Transactions on Visualization and Computer Graphics 15 (2009), 1433-1440.

[9] H. Chen, S. Zhang, W. Chen, H. Mei, J. Zhang, A. Mercer, R. Liang, H. Qu, Uncertainty-aware Multidimensional Ensemble Data Visualization and Exploration, IEEE Transactions on Visualization and Computer Graphics, DOI 10.1109/TVCG.2015.2410278, 2015.

[10] B.J. Crain, S. Mori, V.P. Chacko, P. Zijl, Three-dimensional tracking of axonal projections in the brain by magnetic resonance imaging, Annals of neurology 45 (1999) 265-269.

[11] A. Daducci et al. Quantitative Comparison of Reconstruction Methods for Intra-Voxel Fiber Recovery From Diffusion MRI, IEEE Transactions on Medical Imaging 33 (2014) 384-399.

[12] M. Descoteaux, E. Angelino, S. Fitzgibbons, R. Deriche, Regularized, Fast and Robust Analytical Q-Ball Imaging, Magnetic Resonance in Medicine 58 (2007), 497-510.

[13] M. Descoteaux, R. Deriche, T.R. Knosche, A. Anwander, Deterministic and probabilistic tractography based on complex fibre orientation distributions, IEEE Transactions on Medical Imaging 28 (2009), 269-286.

[14] M. Ester, H.P. Kriegel, J. Sander, X. Xu, A density-based algorithm for discovering clusters in large spatial databases with noise, In: Proceedings of the Second International Conference on Knowledge Discovery and Data Mining, 1996, pp.226-231.

[15] Y. Feng, P. Savajiev, Y. Rathi, M. Quan, Z. Wang, C.F. Westin, A Swarm Tracking Approach for Stochastic White Matter Tractography, IEEE International Symposium on Biomedical Imaging:From Nano to Macro (2011) 803-807.

[16] O. Friman, G. Farneback, C.-F. Westin, A Bayesian Approach for Stochastic White Matter Tractograph, IEEE Transactions on Medical Imaging 25 (2006) 965-978.

[17] A. Ghosh, E. Tsigaridas, B. Mourrain, R. Deriche, A polynomial approach for extracting the extrema of a spherical function and its application in diffusion MRI, Medical image analysis 17 (2013) 503-514.

[18] S. Jbabdi, M.W. Woolrich, J.L. Andersson, T.E. Behrens, A Bayesian framework for global tractography, NeuroImage 37 (2007) 116-129.

[19] B. Jeurisse, A. Leemans, J.D. Tournier, D.K. Jones, J. Sijbers, Estimating the number of fiber orientations in diffusion MRI voxels: a constrained spherical deconvolution study, In: Proceedings 18th Scientific Meeting, International Society for Magnetic Resonance in Medicine, Stockholm, 2010, pp.573.

[20] R. Jianu, C. Demiral, D.H. Laidlaw, Exploring brain connectivity with two-dimensional neural maps, IEEE Transactions on Visualization and Computer Graphics 18 (2012) 978-987.

[21] F. Jiao, Y. Gur, C.R. Johnson, S. Joshi, Detection of crossing white matter fibers with high-order tensors and rank-k decompositions, In: Information Processing in Medical Imaging, Springer, 2011, pp.538-549.

[22] F. Jiao, J.M. Philiips, Y. Gur, C.R. Johnson, Uncertainty visualization in HARDI based on ensembles of ODFs, In: IEEE Pacific Visualization Symposium , 2012, 
pp.193-200.

[23] E. Kaden, T.R. Knösche, A. Anwander, Parametric spherical deconvolution: Inferring anatomical connectivity using diffusion MR imaging, NeuroImage 37 (2007) 474-488.

[24] D. Keim, M.C. Hao, J. Ladisch, M. Hsu, U. Dayal, Pixel bar charts: a new technique for visualizing large multi-attribute data sets without aggregation, In: IEEE Symposium on Information Visualization, San Diego, 2001, pp.113-120.

[25] G. Kindlmann, C.F. Westin, Diffusion Tensor Visualization with Glyph Packing, IEEE Transactions on Visualization and Computer Graphics 12 (2006) 1329-1336.

[26] L. Lacerda. HARDI Methods: tractography reconstructions and automatic parcellation of brain connectivity, Ph.D. thesis, Universidade de Lisboa, 2012.

[27] A.M. MacEachren, A. Robinson, S. Hopper, S. Gardner, R. Murray, M. Gahegan, E. Hetzle, Visualizing geospatial information uncertainty: what we know and what we need to know, Cartography and geographic Information Science 32 (2005) 139-160.

[28] M. Maddah, W.E. Grimson, S.K. Warfield, W.M. Wells, A unified framework for clustering and quantitative analysis of white matter fiber tracts, Medical Image Analysis 12 (2008) 191-202.

[29] J.F. Mangin, P. Fillard, Y. Cointepas, D. Le Bihan, V. Frouin, Toward global tractography, NeuroImage 80 (2013) 290-296.

[30] J.G. Malcolm, M.E. Shenton, Y. Rathi, Filtered multitensor tractography, IEEE Transactions on Medical Imaging 29 (2010) 1664-1675.

[31] M. Hlawitschka, S. Eichelbaum, G. Scheuermann, Fast and Memory Efficient GPU-Based Rendering of Tensor Data, In: Proceedings of the IADIS International Conference on Computer Graphics and Visualization, 2008, pp. 28-30.

[32] B. Moberts, A. Vilanova, J.J. van Wijk, Evaluation of fiber clustering methods for diffusion tensor imaging. In: Proceedings of IEEE Visualization, 2005, pp. 65-72.

[33] L.J. O’Donnell, C.-F. Westin, Automatic tractography segmentation using a high-dimensional white matter atlas, IEEE Transactions on Medical Imaging 26 (2007) 1562-1575.

[34] E. Özarslan, T.M. Shepherd, B.C. Vemuri, S.J. Blackband, T.H. Mareci, Resolution of complex tissue microarchitecture using the diffusion orientation transform (DOT), NeuroImage 31 (2006) 1086-1103.

[35] F.J. Post, T. van Walsum, F.H. Post, D. Silver, Iconic techniques for feature visualization, In: Proceedings of IEEE Visualization, 1995, pp.288-295.

[36] K. Potter, P. Rosen, C.R. Johnson, From quantification to visualization: a taxonomy of uncertainty visualization approaches, In: Uncertainty quantification in scientific computing, ser. IFIP Advances in Information and Communication Technology Series, 2012, 226-249.

[37] Y. Rathi, O. Michailovich, S. Bouix, M. Shenton, Directional functions for orientation distribution estimation, Medical image analysis, 13 (2009) 432-444.

[38] Y. Rathi, M. Kubicki, S. Bouix, C.-F. Westin, J. Goldstein, L. Seidman, R. Mesholam-Gately, R.W. MecCarley, M.E. Shenton, Statistical analysis of fiber bundles using multi-tensor tractography: application to first-episode schizophrenia, Magnetic Resonance in Medicine 29 ( 2011) 507-515. 
[39] T. Schultz , G.L. Kindlmann, Open-box spectral clustering: applications to medical image analysis, IEEE Transactions on Visualization and Computer Graphics 19 (2013) 2100-2108.

[40] D.W. Shattuck, M.C. Chiang, M. Barysheva, K.L. McMahon, G.I. de Zubicaray, M. Meredith, M.J. Wright, A.W. Toga, P.M. Thompson, Visualization Tools for High Angular Resolution Diffusion Imaging, Proceeding Medical Image Computing and Computer Assisted Intervention 2008 298-305.

[41] A. Tsai, C.-F Westin, A.O. Hero, A.S. Willsky, Fiber tract clustering on manifolds with dual rooted-graphs, In: Proceedings of IEEE Computer Vision and Pattern Recognition, 2007, 1-6.

[42] J.D. Tournier, F. Calamante, A. Connelly, Robust determination of the fibre orientation distribution in diffusion MRI: Non-negativity constrained super-resolved spherical deconvolution, NeuroImage 35 ( 2007) 1459-1472.

[43] D.S. Tuch, D. Solomon, Diffusion MRI of complex tissue structure, Ph.D. thesis, Harvard University and Massachusetts Institute of Technology, 2002.

[44] D.S. Tuch, Q-ball imaging, Magnitic Resonance in Medicine, 52 (2004) 1358-1372.

[45] M. van Almsick, T.H. Peeters, V. Prckovska, A. Villanova, R.B. Ter Haar, GPU-Based Ray-Casting of Spherical. Functions Applied to High Angular Resolution Diffusion Imaging, IEEE Transactions on Visualization and Computer Graphics 17 (2011) 612-625.

[46] Q. Wang, P. T. Yap, G. Wu, D. Shen, Application of Neuroanatomical Features to Tractography Clustering, Hum Brain Mapp 34 (2013) 2089-2102.

[47] V.J. Wedeen, R.P. Wang, J.D. Schmahmann, T. Benner, W.Y. Tseng, G. Dai, D.N. Pandya, P. Hagmann, H. D'Arceuil, A.J. de Crespigny, Diffusion spectrum magnetic resonance imaging (DSI) tractography of crossing fibers, NeuroImage 41 (2008) 1267-1277.

[48] Y.T. Weldeselassie, A. Barmpoutis, M. Atkins, Symmetric positive semi-definite cartesian tensor fiber orientation distributions (CT-FOD), Medical Image Analysis 16 (2012) 1121-1129.

[49] V. Wiens, L. Schlaffke, T. Schmidt-Wilcke, T. Schultz1, Visualizing Uncertainty in HARDI Tractography Using Superquadric Streamtubes, Eurographics Conference on Visualization (2014)

[50] S. Zhang, S. Correia, D.H. Laidlaw, Identifying white-matter fiber bundles in DTI data using an automated proximity-based fiber-clustering method, IEEE Transactions on Visualization and Computer Graphics 14 (2008) 1044-1053.

[51] F. Zhang, E.R. Hancock,G. Gerig, Probabilistic white matter fiber tracking using particle filtering and von Mises-Fisher sampling, Medical Image Analysis 13 (2009) $5-18$. 